\title{
Risk factors for adjacent segment disease after lumbar fusion
}

\author{
Choon Sung Lee $\cdot$ Chang Ju Hwang \\ Sung-Woo Lee $\cdot$ Young-Joon Ahn · Yung-Tae Kim • \\ Dong-Ho Lee $\cdot$ Mi Young Lee
}

Received: 24 July 2008/Revised: 19 April 2009/Accepted: 1 June 2009/Published online: 16 June 2009

(C) Springer-Verlag 2009

\begin{abstract}
The incidence of adjacent segment problems after lumbar fusion has been found to vary, and risk factors for these problems have not been precisely verified, especially based on structural changes determined by magnetic resonance imaging. The purpose of this retrospective clinical study was to describe the incidence and clinical features of adjacent segment disease (ASD) after lumbar fusion and to determine its risk factors. We assessed the incidence of ASD in patients who underwent lumbar or lumbosacral fusions for degenerative conditions between August 1995 and March 2006 with at least a 1-year followup. Patients less than 35 years of age at the index spinal fusion, patients with uninstrumented fusion, and patients who had not achieved successful union were excluded. Of the 1069 patients who underwent fusions, 28 (2.62\%) needed secondary operations because of ASD and were included in this study. In order to identify the risk factors, we matched a disease group and a control group. The disease group consisted of 26 of the 28 patients with ASD, excluding the 2 patients for whom we did not have initial MRI data. Each patient in the disease group was matched
\end{abstract}

C. S. Lee · C. J. Hwang $(\bowtie) \cdot$ Y.-T. Kim ·

D.-H. Lee - M. Y. Lee

Department of Orthopaedic Surgery, Asan Medical Center, College of Medicine, University of Ulsan,

388-1, Poongnap-dong, Songpa-gu, Seoul 138-736, Korea

e-mail: baski47@gmail.com

S.-W. Lee

Department of Orthopaedic Surgery,

Seoul Chuk Spine Hospital, 16-512, Jeoungneung-dong,

Seongbook-gu, Seoul 136-841, Korea

Y.-J. Ahn

Department of Orthopaedic Surgery,

National Police Hospital, Seoul, Korea by age, sex, fusion level and follow-up period with a control patient. The assumed risk factors included disc and facet degeneration, instability, listhesis, rotational deformity, and disc wedging. The mean age of the 28 patients with ASD requiring surgical treatment was 58.4 years, which did not differ significantly from that of the population in which ASD did not develop (58.2 years, $p=0.894)$. Of the 21 patients who underwent floating fusion, only 1 developed distal ASD. Facet degeneration was a significant risk factor $(p<0.01)$ on logistic regression analysis. The incidence of distal ASD was much lower than that of proximal ASD. Pre-existing facet degeneration may be associated with a high risk of adjacent segment problems following lumbar fusion procedures.

Keywords Adjacent segment - Degeneration . Lumbar fusion · Risk factor

\section{Introduction}

Although efforts to preserve segmental motion during spinal surgery are now increasing, spinal fusion remains a standard method of surgical treatment for deformity, trauma, and degenerative disorders. Fusion and clinical success rates have increased due to improvements in instrumentation and bone graft material. In contrast, numerous complications and problems of fusion surgery have been reported, with adjacent segment disease (ASD) being one of the most important. Abnormal loading and increased mobility in adjacent segments may explain the development of ASD, but it is still unclear whether it is caused by fusion sequelae or is the result of natural degeneration $[3,6,11,15,18,24]$. There have also been controversies about the exact incidence of ASD and its risk 
factors. It is evident that the number of surgical interventions for ASD will increase as more spinal fusions are performed. To date, however, only a few reports on the results of revision surgery for ASD have been published [1, 4, 22].

The authors described the incidence and clinical features of ASD after lumbar fusion based on the past 11-year experience, and tried to elucidate the risk factors for ASD, as determined by lumbosacral plain radiographs and magnetic resonance imaging (MRI).

\section{Materials and methods}

We retrospectively evaluated the results of 1069 instrumented lumbar or lumbosacral fusions performed at our institution for the treatment of degenerative conditions, with at least a 1-year follow-up period, between August 1995 and March 2006. Patients less than 35 years of age at the time of index spinal fusion, patients who underwent uninstrumented fusion, and patients who did not achieve successful union were excluded. The initial diagnosis included spinal stenosis, isthmic and degenerative spondylolisthesis, lumbar degenerative kyphosis, degenerative disc disease and disc herniation. There was no limitation in the type of fusion. Of these 1069 patients, 28 (2.62\%) required and underwent revision surgery due to the development of ASD. ASD was defined as a condition in which a patient showed the relief of symptoms for at least 6 months after the index operation, the newly developed symptoms were compatible with the lesions in adjacent segments demonstrated in radiological images, and the patient had revision surgery for that problem.

Medical records and radiological studies of the ASD patients were retrospectively reviewed to record age, initial diagnosis, type of fusion, neurological abnormalities, the interval between the index and revision procedures, and features of ASD.

To identify the risk factors for ASD, we selected a control group from the fusion population, matched $1: 1$ to the ASD patients by age, sex, fusion level and follow-up period. We assumed that risk factors on MRI would include disc and facet degeneration of the adjacent segment, and risk factors on plain radiographs would include instability, listhesis, rotational deformity, and disc wedging. Therefore, our control patients were chosen only from those with available preoperative standing and flexion-extension radiographs and MRI. The disease group consisted of 26 of the 28 patients with ASD, excluding 2 patients whose initial MRIs were not available. From the patients without ASD, we chose 26 matched by age, gender, fusion level, and follow-up period and assigned to the control group. For the establishment of the control group, we first identified all patients whose gender and fusion level were identical to each patient in the disease group, then selected patients with an age difference of less than 5 years, and finally selected the patient mostly closely matched by follow-up period. The difference in follow-up period between the disease group and the control group was less than one year in all matched cases.

Disc degeneration on MRI was rated from grade 1 to grade 5 using the classification system of Pfirmann et al. [21], and facet joint degeneration was rated from grade 1 to grade 4 according to the criteria of Weishaupt et al. [27]. Two spine surgeons independently reviewed all lumbosacral MRI images of the patients in both groups taken within 6 months before index fusion surgery. The readers, who were blinded to all clinical information, graded degeneration of the disc and facet joints in the proximal or distal adjacent segment in which ASD later developed. Measurements were performed twice for each patient, with the average grade used for statistical analyses. For each matched patient in the control group, the same segment was graded as that of the matched patient in the disease group. If ASD developed in two consecutive segments, data from only the segment nearest fusion site was included in risk factor analysis. Four assumed risk factors on plain radiographs were also measured in the same segment as in MRI: (a) instability, defined as $>3 \mathrm{~mm}$ anterior or posterior translation and $>10^{\circ}$ angulation in flexion/extension lateral radiographs; (b) listhesis, defined as $>3 \mathrm{~mm}$ anterior, posterior, or lateral translation in standing anteroposterior and lateral radiographs; (c) rotational deformity, defined as a $>5^{\circ}$ rotation angle difference between the upper and lower vertebrae measured by Perdriolle's torsionmeter [20] in standing anteroposterior radiograph; and (d) disc wedging, defined as a $>5^{\circ}$ angle between the inferior endplate of the upper vertebra and the superior endplate of the lower vertebra in standing anteroposterior radiograph.

Interobserver and intraobserver agreement in MRI grading was evaluated by kappa statistics. Logistic regression analysis was used to analyze the assumed risk factors with backward elimination, in which variables with significance level of more than 0.25 are removed. Confidence interval of odds ratio was 95\%. Fisher's exact test was also performed for the four categorical variables on plain radiographs. Student's $t$ test was used to compare average ages at index fusion surgery between the patients with ASD (28 patients) and the patients without ASD (1,041 patients) in the fusion population.

\section{Results}

The incidence and clinical features of ASD were based on data from the 28 patients who required and underwent 
revision surgery. Risk factors were identified based on data from 26 patients in the disease group and 26 in the control group.

Incidence and age

Of the 1,069 patients who underwent lumbar or lumbosacral fusion, $28(2.62 \%)$ underwent revision surgery for ASD. The average age of these ASD patients at the time of index fusion surgery was 58.4 years (range, 46-76 years), which showed no significant difference from that of the 1,041 patients without ASD, 58.2 years $(p=0.894)$. The average interval between the index and revision surgery was 52.3 months (range, 9-125 months).
Diagnosis and clinical features (Table 1)

Degenerative spondylolisthesis was the most common initial diagnosis (19 patients). A single level was fused in 11 patients, two levels in 9 patients, and three levels in 7 patients. In one patient (case 3), spinal stenosis developed in the intervening segment after skip fusions at L2-3 and L4-5. Eight patients underwent posterolateral fusion (PLF) alone; seven underwent posterior lumbar interbody fusion (PLIF) alone, and eight underwent combined PLF and PLIF, each at a different level. In the remaining five patients, PLF was combined with PLIF or anterior lumbar interbody fusion (ALIF) in one segment.

Table 1 Profiles of patients with ASD

\begin{tabular}{|c|c|c|c|c|c|c|c|}
\hline $\begin{array}{l}\text { Case } \\
\text { No. }\end{array}$ & $\begin{array}{l}\text { Age/ } \\
\text { sex }\end{array}$ & Initial diagnosis & $\begin{array}{l}\text { Initial } \\
\text { fusion }\end{array}$ & $\begin{array}{l}\text { Fusion } \\
\text { technique }\end{array}$ & $\begin{array}{l}\text { Interval to } \\
\text { revision (months) }\end{array}$ & $\begin{array}{l}\text { Motor } \\
\text { weakness }\end{array}$ & ASD \\
\hline 1 & $57 / \mathrm{F}$ & DSL L4-5 & L4-5 & PLF and PLIF & 33 & No & SS L2-4 \\
\hline 2 & $60 / \mathrm{F}$ & SS L2-4, DSL L4-5 & $\mathrm{L} 2-5$ & PLF, PLIF & 14 & Yes & SS and HIVD T12-L1-2 \\
\hline 3 & $69 / \mathrm{F}$ & HIVD L2-3, DSL L4-5 & L2-3, L4-5 & PLF, PLIF & 37 & No & SS L3-4 \\
\hline 4 & $57 / \mathrm{F}$ & SS L3-5 & L3-5 & PLF & 99 & Yes & RL and SS L2-3 \\
\hline 5 & $52 / \mathrm{F}$ & DSL L4-5 & L4-5 & PLIF & 98 & No & AL and SS L3-4 \\
\hline 6 & $65 / \mathrm{M}$ & DSL L3-5, DS,SL L5 & L3-5-S1 & PLIF & 16 & Yes & RL and SS L2-3 \\
\hline 7 & $66 / \mathrm{F}$ & SS L4-5-S1, HIVD L1-2 & L4-5-S1 & PLF & 44 & Yes & RL L2-3, SS L2-4 \\
\hline 8 & $48 / \mathrm{F}$ & DSL L4-5 & L4-5 & PLF and ALIF & 116 & No & AL and SS L3-4 \\
\hline 9 & $58 / \mathrm{F}$ & DSL L3-5 & L3-5 & PLF, PLIF & 16 & Yes & HIVD and SS L5-S1 \\
\hline 10 & $52 / \mathrm{F}$ & SS L4-5-S1 & L4-5-S1 & PLF, PLIF & 9 & No & SS L3-4 \\
\hline 11 & $55 / \mathrm{M}$ & DSL L3-5, SS L3-5-S1 & L3-5-S1 & PLIF & 11 & Yes & SS L3-4 \\
\hline 12 & $65 / \mathrm{M}$ & SS L2-5 & $\mathrm{L} 2-5$ & PLF, PLIF & 41 & Yes & RL and SS L1-2 \\
\hline 13 & $50 / \mathrm{F}$ & RL and SS L4-5 & L4-5 & PLF & 68 & No & AL and SS L3-4 \\
\hline 14 & $59 / \mathrm{F}$ & SS L4-5 & L4-5 & PLF & 55 & No & SS L2-4, RL L3-4 \\
\hline 15 & $48 / \mathrm{F}$ & DSL L4-5, SS L3-5 & L3-5 & PLF & 76 & No & AL and SS L2-3 \\
\hline 16 & $59 / \mathrm{F}$ & DSL L4-5 & L4-5 & PLF and PLIF & 52 & No & HIVD L2-4, AL L3-4 \\
\hline 17 & $64 / \mathrm{M}$ & SS L3-5 & L3-5 & PLF & 28 & Yes & SS and HIVD L2-3 \\
\hline 18 & $58 / \mathrm{M}$ & DSL L4-5 & L4-5 & PLF & 26 & No & HIVD and SS L3-4 \\
\hline 19 & $53 / \mathrm{M}$ & DSL L4-5 & L4-5 & PLF and ALIF & 125 & No & RL and HIVD L2-3, SS L2-4 \\
\hline 20 & $46 / \mathrm{F}$ & DSL L4-5, SS L3-5 & L3-5 & PLF, PLIF & 80 & No & AL and SS L2-3 \\
\hline 21 & $76 / \mathrm{M}$ & DSL L5-S1 & L5-S1 & PLIF & 50 & No & AL and SS L4-5 \\
\hline 22 & $61 / \mathrm{M}$ & ISL L5-S1, SS L4-5-S1 & L4-5-S1 & PLF, PLIF & 55 & No & RL and SS L2-4 \\
\hline 23 & $51 / \mathrm{F}$ & DSL L3-4, SS L3-5 & L3-5 & PLF, PLIF & 35 & No & AL, SS and HIVD L2-3 \\
\hline 24 & $60 / \mathrm{F}$ & DSL L3-4, RL L4-5 & L3-5 & PLIF & 59 & No & AL with kyphosis L1-2, LL L2-3, DS \\
\hline 25 & $53 / \mathrm{F}$ & DSL L4-5 & L4-5 & PLF & 79 & No & AL and SS L3-4 \\
\hline 26 & $70 / \mathrm{M}$ & DSL L4-5 & L4-5 & PLF and PLIF & 50 & No & SS L3-4 \\
\hline 27 & $64 / \mathrm{F}$ & DSL L4-5, SS L4-5-S1 & L4-5-S1 & PLIF & 66 & No & SS L2-4 \\
\hline 28 & $60 / \mathrm{M}$ & SS L2-S1 & L2-S1 & PLF & 27 & Yes & AL, SS and HIVD L1-2, VCF L1 \\
\hline
\end{tabular}

$D S L$ degenerative spondylolisthesis, $S S$ spinal stenosis, $H I V D$ herniated intervertebral disc, $D S$ degenerative scoliosis, $S L$ spondylolysis, $R L$ retrolisthesis, $I S L$ isthmic spondylolisthesis, $P L F$ posterolateral fusion, $P L I F$ posterior lumbar interbody fusion, ALIF anterior lumbar interbody fusion, $A S D$ adjacent segment disease, $A L$ anterolisthesis, $L L$ lateral listhesis, $V C F$ vertebral compression fracture 

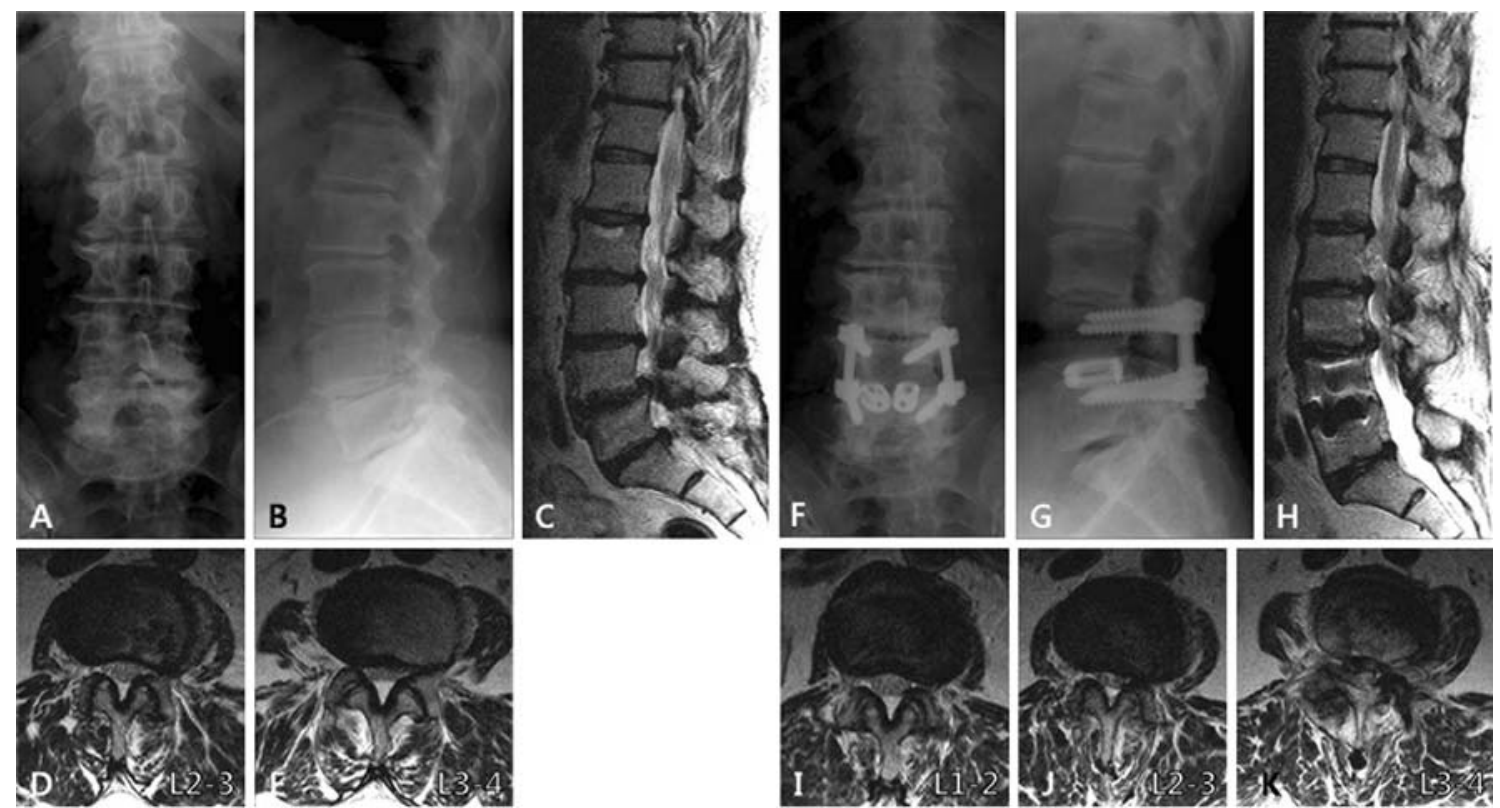

Fig. 1 Imaging studies of case 1. This patient had degenerative spondylolisthesis at L4-5 (a, b and c). Initial radiographs and MRI showed rotational deformity at L3-4, disc wedging at L2-3, and grade 4 disc degeneration at L2-3, L3-4 and L5-S1 (c). Facet degeneration was grade 2 at L2-3 (d) and grade 1 at L3-4 (e). PLIF

was performed at L4-5. Adjacent segment disease developed at L2-3 and L3-4 after 33 months (f, $\mathbf{g}$ and $\mathbf{h})$. Central spinal stenosis was aggravated at L2-3 (j) and L3-4 (k). Facet joints were intact at L1-2 (i). Revision surgery was performed from L2 to L5. Note that the L5-S1 segment does not show any deterioration of degeneration

In general, back pain and radiating leg pain were relatively severe, although they were not quantitated. Motor weakness greater than one grade developed in the major leg muscles of nine patients $(32.1 \%)$. Two of these patients normalized and four patients improved after revision surgery. All patients with ASD had spinal stenosis with neural encroachment, accompanied by disc herniation in 8 patients, spondylolisthesis in 11 patients, and retrolisthesis in 7 patients. Degenerative lumbar kyphoscoliosis with lateral listhesis in the upper adjacent segment developed after L3-5 fusion in one patient (case 24). L5-S1 was initially fused in 7 patients, while 21 patients underwent floating fusion. Of the latter, only one (case 10, L5-S1) developed distal ASD. Of the 27 patients with proximal ASD, 9 (33.3\%) showed degenerative changes in two consecutive segments (Fig. 1).

\section{Risk factors (Table 2)}

Interobserver agreement for grading of disc and facet joints in initial MRI was substantial and fair with weighted kappa coefficients of 0.65 and 0.37 , respectively $(0.00$ $0.20=$ slight,$\quad 0.21-0.40=$ fair,$\quad 0.41-0.60=$ moderate, $0.61-0.80=$ substantial, $\quad 0.80-1.00=$ excellent $\quad$ [14] . Intraobserver agreements were substantial for both variables, with kappa values of 0.76 and 0.74 , respectively. Average degenerative disc changes were grade 3.58 in the disease group, and grade 3.23 in the control group. Facet

arthrosis was grade 1.58 in the disease group and grade 0.92 in the control group. Of the assumed risk factors on plain radiographs, instability was observed in six patients of the disease group and four patients of the control group; listhesis in seven and three patients, respectively; rotational deformity in seven and two patients, respectively; and disc wedging in four and two patients, respectively. All six variables had higher average grade or higher incidence in the disease group, but only degeneration of facet joints on initial MRI was found to be a statistically significant risk factor for ASD by logistic regression analysis $(p<0.01)$. The other factors, including disc degeneration, did not differ significantly between the two groups. Fisher's exact

Table 2 Risk factors

\begin{tabular}{lrrrl}
\hline Risk factors & \multicolumn{2}{l}{ Logistic regression } & $\begin{array}{l}\text { Fisher's } \\
\text { exact } \\
\end{array}$ \\
\cline { 2 - 4 } & $p$ value & $\begin{array}{l}\text { Odds } \\
\text { ratio } \\
p \text { value }\end{array}$ & $\begin{array}{l}95 \% \\
\text { Confidence } \\
\text { interval }\end{array}$ & \\
\hline Disc degeneration & 0.17 & 0.48 & $0.17-1.38$ & \\
Facet degeneration & $<0.01$ & 0.01 & $<0.01-0.12$ & \\
Instability & 0.70 & 0.63 & $0.06-6.46$ & 0.73 \\
Listhesis & 0.41 & 0.38 & $0.04-3.75$ & 0.29 \\
Rotational deformity & 0.83 & 0.74 & $0.05-11.40$ & 0.14 \\
Disc wedging & 0.63 & 1.81 & $0.16-20.61$ & 0.67 \\
\hline
\end{tabular}


test also showed no statistical significance for the four categorical variables.

\section{Discussion}

Hilibrand and Robbins [12] used the term 'adjacent segment degeneration' to describe radiographic changes seen at levels adjacent to a previous spinal fusion procedure, which do not necessarily correlate with any clinical findings. 'Adjacent segment disease' has been used to designate the development of new clinical symptoms that are compatible with radiographic changes adjacent to the level of a previous spinal fusion. Although studies on the adjacent segment problem are now in active progress, but most of them have utilized radiographic findings as a diagnostic tool rather than for symptomatology [2, 5, 7-10, 19]. However, we suggest that discussion about the adjacent segment changes irrelevant to clinical findings is not so meaningful. Therefore, ASD was defined as a condition in which a patient showed the relief of symptoms for a period of time and then developed new symptoms, accompanied by compatible lesions in adjacent segments on MRI, and subsequently underwent surgery for that problem.

There continues to be debate over the exact incidence of adjacent segment change and whether it is related to actual clinical symptoms. This is likely due to the diversity of ASD definitions or criteria. Park et al. [18] reviewed the results of 56 studies, and found that the incidence of ASD ranged from 8 to $100 \%$ when using only radiographic criteria, whereas the incidence of symptomatic ASD was much lower, ranging from 5.2 to $18.5 \%$. There are few reports of the incidence of adjacent segment problems requiring revision surgery. Aiki et al. [1] performed reoperations for symptomatic adjacent segment stenosis in $7.7 \%$ of patients who had undergone posterior lumbar fusion after at least a 2-year follow-up. Gillet [10] reported that $20 \%$ of posterolateral fusion patients needed a second operation for adjacent motion segment alteration after a minimum of 5 years. According to the survival analysis conducted by Ghiselli et al. [8], the rates of symptomatic degeneration requiring surgery at adjacent segments were $16.5 \%$ at 5 years, and $36.1 \%$ at 10 years after the index fusion. New disease at an adjacent level thus developed at a rate of $3.9 \%$ per year. In our study, revision operations were warranted in $2.62 \%$ of the 1,069 lumbar or lumbosacral fusion patients after at least one year, showing relatively low incidence. However, the incidence is expected to increase with longer follow-up.

The interval between the index and revision surgery has been reported to range from 5.2 to 7.1 years $[1,4,22]$. The comparatively short interval in this study (4.4 years, 52.3 months) may be due to differences in type of fusion and patient population. The time period for the development of radiographic changes or clinical symptoms would be even shorter than that for revision surgery. For example, Etebar and Cahill [7] reported that symptomatic ASD occurred within 1 year from the index operation in 8 of 18 patients. Interestingly, a female patient in this study (case 10) also presented newly developed radiating leg pain and neurogenic claudication only 5 months after surgery. She had been symptom-free since the index L4-S1 fusion surgery. MRI revealed aggravation of spinal stenosis caused by disc herniation and facet hypertrophy at L3-4. In particular, this patient had a trefoil spinal canal. This is the reason why we chose 1 year as the minimum follow-up period for the fusion population.

It is unclear whether the distal L5-S1 segment should be included in fusion levels when floating fusion, including L4-5, is needed. The number of reports on this problem, however, is unexpectedly scanty. Survivorship analysis by Ghiselli et al. [9] has shown that the 10-year survival rate of the L5-S1 segment after isolated L4-5 fusion was $90 \%$. Miyakoshi et al. [16] suggested that the L5-S1 segment does not have to be routinely included in fusion, even in the presence of degenerative changes, because the clinical outcome of L4-L5 fusion is not affected by narrowing of the L5-S1 disc. We also found that the incidence of distal ASD was much lower than that of proximal ASD. The exact statistical data have not been collected, but several other clinical studies have demonstrated similar tendencies $[1,2,5,7]$. This may be due to the recessed position of the L5-S1 segment within the pelvis restrained by iliolumbar ligaments [9]. Moreover, the possibility of facet injury by pedicle screw insertion is relatively low in the inferior adjacent segment as compared to the superior adjacent segment [2]. The wide cross-sectional area of the spinal canal at the L5-S1 level may also contribute to this result. Although additional studies are required, including determination of the differences in biomechanical changes induced by fusion between the proximal and distal adjacent segments, we recommend the preservation of the L5-S1 segment as long as there are no serious degenerative changes or neural encroachment.

Many efforts have been made to find risk factors for ASD in order to predict and prevent this condition, with numerous factors suspected as culprits including patient factors such as age, gender, or bone mineral density; anatomical predisposition of the lamina and facet joints; preoperative condition of the disc and facet joints; number of fused segments; type of fusion and instrumentation; and coronal or sagittal alignment $[1,2,5,7,8,10,13,17,18$, 23, 26, 28]. Since Aota et al. [2] indicated that the incidence of post-fusion instability at the adjacent segments was far higher in older ( $>55$ years) than in younger patients, patient age has been regarded as a major risk 
factor. These findings, however, have been negated by some recently published articles $[1,7,17]$, and this study also demonstrated that the average age of the ASD patients did not differ significantly between patients with and without ASD.

A better determination of fusion levels during operative planning requires the ability to predict whether preexisting disc or facet degeneration will cause ASD in the future. Because degenerative changes seldom localize in the segments requiring decompression during surgery on patients with lumbar degenerative disease, determination of fusion levels is always confusing. Throckmorton et al. [25] claimed that no benefit was derived from including adjacent segments with disc degeneration on preoperative MRI in lumbar fusion because degenerated adjacent segments did not appear to adversely affect clinical outcomes. Another study performed to identify the risk factors for ASD reported no statistically significant relationship with adjacent segment disc degeneration on MRI [17]. To our knowledge, however, no studies have reported on the correlation between facet degeneration and ASD. Among the six assumed risk factors in our study, only degenerative change of facet joints detected on MRI was statistically significant, although the grade and incidence of other variables were higher in the disease group.

Grading of facet arthrosis on MRI encompasses the problem of low interobserver reliability. The current authors observed only fair interobserver agreement. Weishaupt et al., who devised the grading system used in this study, concluded that computed tomography (CT) is the radiologic method of choice in the diagnosis of facet joint degeneration because of this limitation [27]. Accordingly, evaluation of facet status by $\mathrm{CT}$ will be a good alternative if facet degeneration at adjacent levels is of concern in the determination of fusion levels. There was a statistical significance in the odds ratio of the facet degeneration between the disease and control groups in this study, but the average difference was less than one grade. Therefore, fusion level cannot be exclusively determined by this factor, and other variables should be considered.

\section{Conclusions}

Although we observed a relatively low incidence of ASD after lumbar or lumbosacral fusion requiring surgery, patients with ASD had relatively severe symptoms and frequent neurological abnormalities. In determining fusion levels, it would be desirable to save the distal adjacent segment unless it shows significant degenerative changes or neural encroachment because the incidence of distal ASD is much lower than that of proximal ASD in floating fusion. Preexisting facet degeneration may be associated with a high risk of adjacent segment problems.

Acknowledgments The authors thank Keunpyo Kim, PhD (MedImmune, Gaithersburg, MD) and Joseph Hong, DO (Rothman Institute, Philadelphia, PA) for their help in the preparation of this manuscript.

\section{References}

1. Aiki H, Ohwada O, Kobayashi H, Hayakawa M, Kawaguchi S, Takebayashi T, Yamashita T (2005) Adjacent segment stenosis after lumbar fusion requiring second operation. J Orthop Sci 10:490-495. doi:10.1007/s00776-005-0919-3

2. Aota Y, Kumano K, Hirabayashi S (1995) Postfusion instability at the adjacent segments after rigid pedicle screw fixation for degenerative lumbar spinal disorders. J Spinal Disord 8:464-473. doi:10.1097/00002517-199512000-00008

3. Bastian L, Lange U, Knop C, Tusch G, Blauth M (2001) Evaluation of the mobility of adjacent segments after posterior thoracolumbar fixation: a biomechanical study. Eur Spine J 10:295-300. doi:10.1007/s005860100278

4. Chen WJ, Lai PL, Niu CC, Chen LH, Fu TS, Wong CB (2001) Surgical treatment of adjacent instability after lumbar spine fusion. Spine 26:E519-E524. doi:10.1097/00007632-20011115000024

5. Chou WY, Hsu CJ, Chang WN, Wong CY (2002) Adjacent segment degeneration after lumbar spinal posterolateral fusion with instrumentation in elderly patients. Arch Orthop Trauma Surg 122:39-43

6. Dekutoski MB, Schendel MJ, Ogilvie JW, Olsewski JM, Wallace LJ, Lewis JL (1994) Comparison of in vivo and in vitro adjacent segment motion after lumbar fusion. Spine 19:1745-1751. doi: 10.1097/00007632-199408000-00015

7. Etebar S, Cahill DW (1999) Risk factors for adjacent-segment failure following lumbar fixation with rigid instrumentation for degenerative instability. J Neurosurg 90:163-169

8. Ghiselli G, Wang JC, Bhatia NN, Hsu WK, Dawson EG (2004) Adjacent segment degeneration in the lumbar spine. J Bone Joint Surg Am 86:1497-1503

9. Ghiselli G, Wang JC, Hsu WK, Dawson EG (2003) L5-S1 segment survivorship and clinical outcome analysis after L4-L5 isolated fusion. Spine 28:1275-1280. doi:10.1097/00007632200306150-00011

10. Gillet P (2003) The fate of the adjacent motion segments after lumbar fusion. J Spinal Disord Tech 16:338-345

11. Ha KY, Schendel MJ, Lewis JL, Ogilvie JW (1993) Effect of immobilization and configuration on lumbar adjacent-segment biomechanics. J Spinal Disord 6:99-105. doi:10.1097/00002517199304000-00002

12. Hilibrand AS, Robbins M (2004) Adjacent segment degeneration and adjacent segment disease: the consequences of spinal fusion? Spine J 4:190S-194S. doi:10.1016/j.spinee.2004.07.007

13. Kumar MN, Baklanov A, Chopin D (2001) Correlation between sagittal plane changes and adjacent segment degeneration following lumbar spine fusion. Eur Spine J 10:314-319. doi: $10.1007 / \mathrm{s} 005860000239$

14. Landis RJ, Koch GG (1977) The measurement of observer agreement for categorical data. Biometrics 33:159-174. doi: $10.2307 / 2529310$

15. Lee CK, Langrana NA (1984) Lumbosacral spinal fusion. A biomechanical study. Spine 9:574-581. doi:10.1097/00007632198409000-00007 
16. Miyakoshi N, Abe E, Shimada Y, Okuyama K, Suzuki K, Sato K (2000) Outcome of one-level posterior lumbar interbody fusion for spondylolisthesis and postoperative intervertebral disc degeneration adjacent to the fusion. Spine 25:1837-1842. doi: 10.1097/00007632-200007150-00016

17. Okuda S, Iwasaki M, Miyauchi A, Aono H, Morita M, Yamamoto $\mathrm{T}$ (2004) Risk factors for adjacent segment degeneration after PLIF. Spine 29:1535-1540. doi:10.1097/01.BRS.0000131417. 93637.9D

18. Park P, Garton HJ, Gala VC, Hoff JT, McGillicuddy JE (2004) Adjacent segment disease after lumbar or lumbosacral fusion: review of the literature. Spine 29:1938-1944. doi:10.1097/01. brs.0000137069.88904.03

19. Pellise F, Hernandez A, Vidal X, Minguell J, Martinez C, Villanueva C (2007) Radiologic assessment of all unfused lumbar segments 7.5 years after instrumented posterior spinal fusion. Spine 32:574-579. doi:10.1097/01.brs.0000256875.17765.e6

20. Perdriolle R (1991) The torsion meter: a critical review. J Pediatr Orthop 11:789

21. Pfirrmann CWA, Metzdorf A, Zanetti M, Hodler J, Boos N (2001) Magnetic resonance classification of lumbar intervertebral disc degeneration. Spine 26:1873-1878. doi:10.1097/00007632200109010-00011

22. Phillips FM, Carlson GD, Bohlman HH, Hughes SS (2000) Results of surgery for spinal stenosis adjacent to previous lumbar fusion. J Spinal Disord 13:432-437. doi:10.1097/00002517200010000-00011
23. Rahm MD, Hall BB (1996) Adjacent-segment degeneration after lumbar fusion with instrumentation: a retrospective study. J Spinal Disord 9:392-400. doi:10.1097/00002517-19961000000005

24. Shono Y, Kaneda K, Abumi K, McAfee PC, Cunningham BW (1998) Stability of posterior spinal instrumentation and its effects on adjacent motion segments in the lumbosacral spine. Spine 23:1550-1558. doi:10.1097/00007632-199807150-00009

25. Throckmorton TW, Hilibrand AS, Mencio GA, Hodge A, Spengler DM (2003) The impact of adjacent level disc degeneration on health status outcomes following lumbar fusion. Spine 28:2546-2550. doi:10.1097/01.BRS.0000092340.24070.F3

26. Umehara S, Zindrick MR, Patwardhan AG, Havey RM, Vrbos LA, Knight GW, Miyano S, Kirincic M, Kaneda K, Lorenz MA (2000) The biomechanical effect of postoperative hypolordosis in instrumented lumbar fusion on instrumented and adjacent spinal stenosis. Spine 25:1617-1624. doi:10.1097/00007632200007010-00004

27. Weishaupt D, Zanetti M, Boos N, Hodler J (1999) MR imaging and CT in osteoarthritis of the lumbar facet joints. Skeletal Radiol 28:215-219. doi:10.1007/s002560050503

28. Wiltse LL, Radecki SE, Biel HM, DiMartino PP, Oas RA, Farjalla G, Ravessoud FA, Wohletz C (1999) Comparative study of the incidence and severity of degenerative change in the transition zone after instrumented versus noninstrumented fusions of the lumbar spine. J Spinal Disord 12:27-33. doi:10.1097/ 00002517-199902000-00004 